\title{
Ocular Oncology and Pathology
}

Ocul Oncol Pathol 2018;4:21-22

DOI: $10.1159 / 000477409$

\section{Inhomogeneous Surface Dose Distributions of ${ }^{106}$ Ru Eye Plaques}

\section{Marion Eichmann}

Fakultät Physik, Technische Universität Dortmund, Dortmund, Germany

This letter is a supplement to the paper of Zaragoza et al. [1]. In this publication, the impact of the heterogeneous distribution of the emitter substance of ${ }^{106} \mathrm{Ru}$ eye plaques on the dose distribution and the treatment planning are exemplarily shown for 2 eye plaques of the CCB and CCA types. The investigated CCB-type eye plaque (serial number 1256) shows inhomogeneities in dose distributions of about $25 \%$ with respect to the ideal homogenous plaque. As a
Received: February 21, 2017

Accepted after revision: May 3, 2017 Published online: June 23, 2017
This project was supported by a grant from the Deutsche Forschungsgemeinschaft (German Research Foundation), Grant No. El 869/1-3.

supplement to [1], 5 other surface dose distributions of the CCBtype eye plaques are shown in this letter (Fig. 1) to illustrate their large variation. For comparison, a Monte Carlo-simulated surface dose distribution of an idealized homogenous eye, plaque is added.

Most published investigations concerning the dosimetry of ${ }^{106} \mathrm{Ru}$ eye plaques contain data for only 1 single eye plaque. For this reason, differences in dose distributions between different publications cannot be easily attributed to methodical differences on the one hand or differences in the heterogeneous distribution of the emitter substance of the eye plaques themselves on the other. Furthermore, this inhomogeneity is one of the reasons why Monte Carlo-simulated depth dose curves [2] differ from measured ones especially regarding absolute doses (Fig. 2). The manufacturer BE-

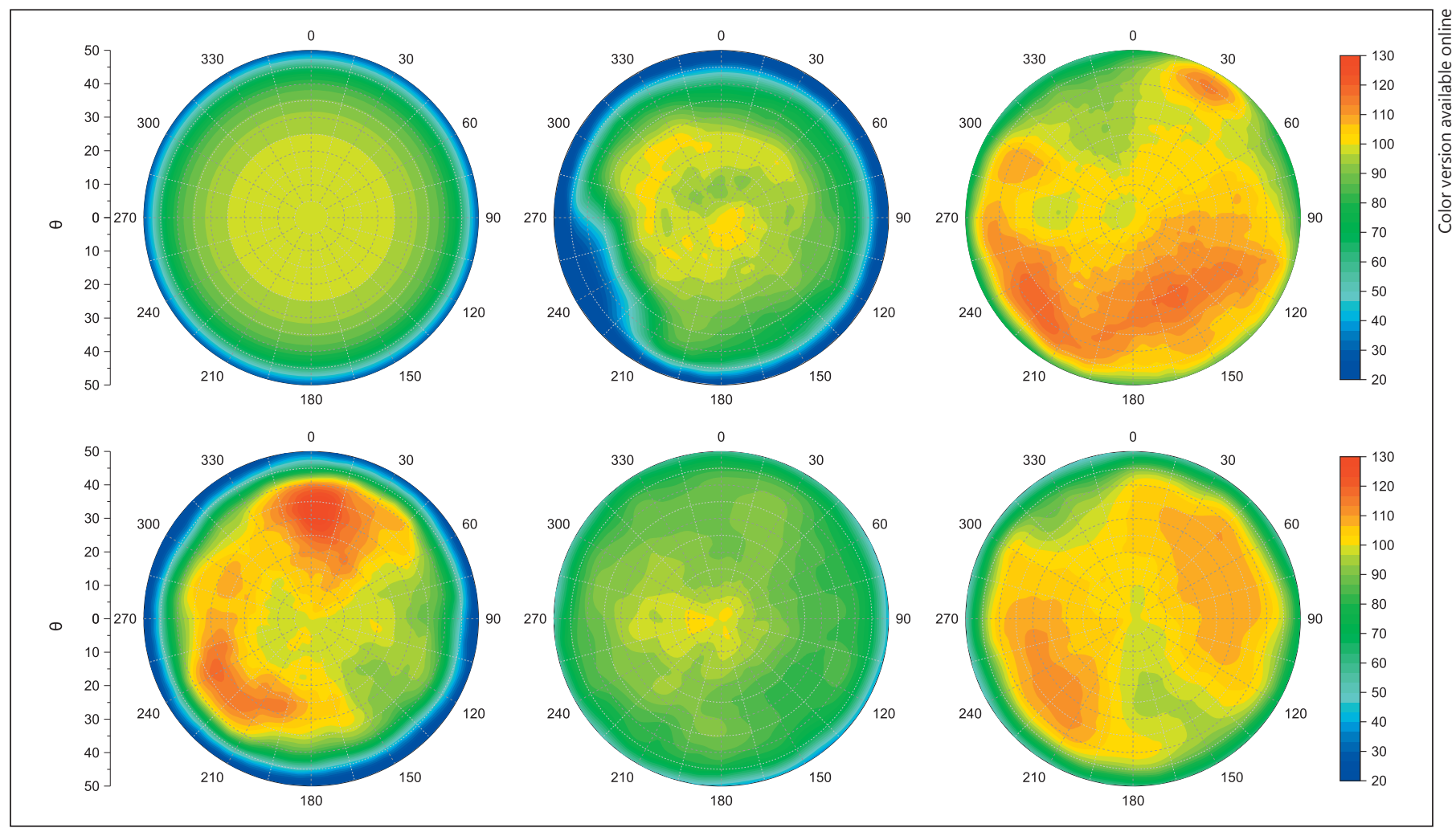

Fig. 1. Surface dose profiles, normalized to the center, of 6 ophthalmic CCB-type plaques (top left to down right; Monte Carlo homogenous plaque, measured CCB type, serial number: 1299, 1767, 1803, 1870, and 1982; data of CCB 1299 are taken from Eichmann [5]).

\section{KARGER}

() 2017 S. Karger AG, Basel

E-Mail karger@karger.com www.karger.com/oop
Marion Eichmann

Fakultät Physik, Experimentelle Physik 5a, Strahlenphysik Otto-Hahn-Strasse 4a

DE-44227 Dortmund (Germany)

E-Mail marion.eichmann@ @u-dortmund.de 


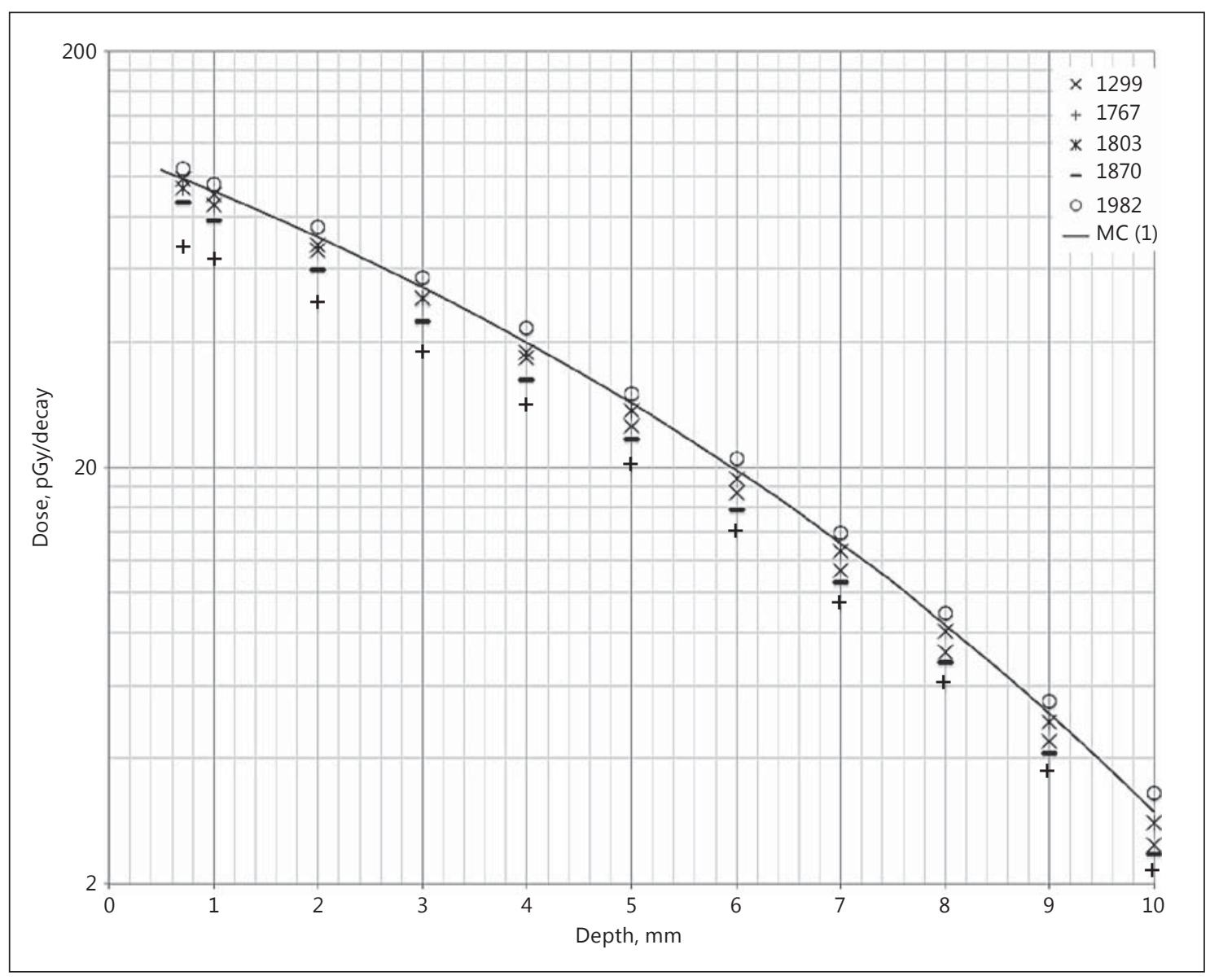

Fig. 2. Dose per activity along the plaque symmetry axis. Comparison between Monte Carlo (MC) simulation [2] and different eye plaques of the CCB type. Data are taken from BEBIG certificates for sealed radioactive sources.

BIG delivers a certificate for every individual eye plaque in which a depth-dose curve and a surface-dose distribution consisting of 33 measuring points are documented. The differences in the heterogeneous distribution of the emitter substance are reflected in the depth dose and by this are sufficiently considered for treatment planning. Alternatively, the 33 measuring points can be entered in the Plaque simulator, a treatment planning software (Eye Physics LLC, Los Alamitos, CA, USA), to estimate the dose distribution of the eye plaque. Caused by only a few measuring points, the variation in surface dose distributions is only illustrated to some degree, and their impact on side effects cannot be fully considered. Thus, at the Technical University of Dortmund, a high-precision dosimetry system was developed, which allows for complete measurements of surface-dose distributions of eye plaques [3,4]. One of these data sets is the basis for the Monte Carlo simulations of inhomogeneous plaques recently shown by Zaragoza et al. [1]. Note the large variation of surface-dose distributions measured close to the plaque surface for 5 randomly chosen CCB-type plaques (Fig. 1). As already said by Zaragoza et al. [1], ${ }^{106} \mathrm{Ru}$ eye plaques are very effective for tumors with a tumor thickness of up to $4 \mathrm{~mm}$, and the impact of the inhomogeneous distribution of the ${ }^{106} \mathrm{Ru}$ eye plaques is negligible. In the case of tumors with tumor thicknesses up to $6 \mathrm{~mm}$, the influence of the inhomogeneous distribution becomes larger. A safe treatment needs a detailed knowledge of the dose distribution of the used eye plaque type to assure complete dose coverage of the entire tumor mass plus a sufficient safety margin and to avoid surpassing the dose tolerated by the sclera.

\section{References}

1 Zaragoza FJ, Eichmann M, Flühs D, Sauerwein W, Brualla L: Monte Carlo estimation of absorbed dose distributions obtained from heterogeneous ${ }^{106} \mathrm{Ru}$ eye plaques. Ocul Oncol Pathol 2017;3:204-209.

2 Sommer H, Ebenau M, Spaan B, Eichmann M: Monte Carlo simulation of ruthenium eye plaques with GEANT4: influence of multiple scattering algorithms, the spectrum and the geometry on depth dose profiles. Phys Med Biol 2017;62:1848-1864.

3 Eichmann M, Flühs D, Spaan B: Development of a high precision dosimetry system for the measurement of surface dose rate distribution for eye applicators. Med Phys 2009;36:4634-4643.

4 Flühs D, Eichmann M, Kirov A: 2D and 3D scintillation dosimetry for brachytherapy; in Beddar S, Beaulieu L (eds): Scintillation Dosimetry. Boca Raton, Taylor \& Francis Inc., 2015.

5 Eichmann M: Entwicklung eines hochpräzisen Dosimetriesystems zur Messung der Oberflächendosisverteilung von Augenapplikatoren. Dissertation, Technische Universität Dortmund, 2010.
Eichmann 